\title{
吡啶类含能化合物的合成研究进展
}

\author{
马丛明 ${ }^{a}$ 刘祖亮 ${ }^{a}$ 许晓娟 ${ }^{b}$ 姚其正 ${ }^{*}, a, c$ \\ ( ${ }^{a}$ 南京理工大学化工学院 南京 210094) \\ ( ${ }^{b}$ 盐城师范学院化学化工学院 盐城 224002) \\ ( ${ }^{c}$ 中国药科大学药学院 南京 210009)
}

\begin{abstract}
摘要 吡啶类化合物在含能材料领域中的研究和应用较为广泛. 从分子结构出发，按照硝基吡啶、吡啶类含能离子盐 和吡啶类含能配合物分类，综述了多种吡啶硝基衍生物的合成，并简单介绍了一些重要的硝基吡啶类含能化合物的特 性及主要应用.
\end{abstract}

关键词 硝基吡啶; 合成; 含能化合物; 进展

\section{Research Progress on the Synthesis of Energetic Pyridines}

\author{
Ma, Congming ${ }^{a} \quad$ Liu, Zuliang ${ }^{a} \quad$ Xu, Xiaojuan ${ }^{b} \quad$ Yao, Qizheng $^{*, a, c}$ \\ ( ${ }^{a}$ School of Chemical Engineering, Nanjing University of Science \& Technology, Nanjing 210094) \\ ( ${ }^{b}$ Department of Chemistry, Yancheng Teachers University, Yancheng 224002) \\ ( ${ }^{c}$ School of Pharmacy, China Pharmaceutical University, Nanjing 210009)
}

\begin{abstract}
Pyridines are widely used in energetic materials. Based on molecular structure, and classified themselves by nitropyridines, pyridine-based energetic salts, pyridine-based energetic complexes, a large number of energetic pyridines are summarized. The characteristics and main applications of some important energetic pyridines are also introduced briefly.

Keywords nitropyridines; synthesis; energetic compounds; progress
\end{abstract}

爆轰性能和安全性是含能化合物在实际应用领域 中首先要考虑的重要因素, 然而, 这恰是 $\mathrm{CHNO}$ 组成的 含能分子存有的本质矛盾, 即爆轰性能提升常伴随产生 感度增加和热稳定性下降等问题 ${ }^{[1 \sim 5]}$. 为此, 设计和合 成新型高能针感含能化合物时可通过以下途径或策略 来改善多方面的性能: (1)以氮杂(芳)环代替苯环作为含 能化合物的基本结构单元, 以提高生成热, 有助于改善 氧平衡; (2)在氮杂(芳)环母核分子结构中引入相邻的氨 基和硝基基团, 利于形成分子内外氢键提高其安全性能 和晶体密度(即提高能量密度); (3)在氮杂(芳)环母核上 增加爆炸性基团或组合上高能结构单元, 如用 $N$-氧化 物代替 $\mathrm{C}-\mathrm{NO}_{2}$, 从而改善氧平衡; 或者连接上苦基单元 等以达到提高分子的热稳定性和针化感度的目标 ${ }^{[6]}$.

与碳环芳香含能化合物相比, 氮杂(芳)环硝基化合 物具有较高的密度和爆速等特性 ${ }^{[7}$ 10], 近期形成研究热 点的多硝基吡啶衍生物就是一类潜在的含能前体, 频频
被选为原料/母核用于新高能钝感炸药的制备 ${ }^{[11,12]}$. 为 满足含能化合物的能量要求, 可在吡啶环上引入多个硝 基、叠氮基或氧化呋咱等爆炸性基团，显然这会带来分 子结构的不稳定性等问题, 因而可通过在吡啶环上增加 氨基形成分子内氢键来提高化合物的特征密度 $\rho_{0}$ 与钝 化感度效应，同时借助分子间氢键升高其堆积系数 $(K$ 值)和化合物的熔点; 也可根据分子结构, 在不影响其 他性能的前提下，通过形成含能化合物的金属盐来提高 分子的耐热性 ${ }^{[13]}$; 另外, 以氮杂含能(芳)环为配体的金 属配合物合成始于 20 世纪 90 年代, 研究发现这类含能 化合物具有良好的安定性和强烈的爆炸性，可用于军 用、民用火工品，爆破器材的起爆药、起爆装药及含能 催化剂等方面 ${ }^{[14]}$.

本文按照吡啶类含能化合物的分子结构类型，综述 了硝基吡啶(主要包括多硝基/叠氮基吡啶、多氨基多硝 基吡啶以及苦基硝基吡啶)、吡啶类含能离子盐及吡啶

* E-mail: qz_yao@163.com

Received February 25, 2014; revised March 14, 2014; published online April 2, 2014.

Project supported by the National Natural Science Foundation of China (No. 21102125).

国家自然科学基金(No. 21102125)资助项目. 
类含能金属配合物的合成, 介绍了其中一些较为重要的 吡啶类含能化合物和配合物的特性和主要应用.

\section{1 硝基吡啶}

1,3,5-三氨基-2,4,6-三硝基苯(TATB)是一种对热、 光、冲击波和机械撞击等外界作用均不敏感的单质炸药, 且是经美国能源部批准的可唯一使用的耐热钝感含能 化合物 ${ }^{[15 ~ 18]}$. 然而, 现有 TATB 的批量生产方法为以均 三氯苯为原料, 经硝化和胺化反应制得, 但存在生产成 本高, 工艺条件苛刻, 副产品不易处理等问题. 因此, 研发一种新型的能量和感度接近 TATB, 生产成本较低 的含能材料迫在眉睫. 近年来, 一些多硝基吡啶及其氮 氧化物因其良好的热稳定性、氧平衡和生成热等受到广 泛关注. 李金山等 ${ }^{[19 ~ 21]}$ 通过密度泛函理论对系列多硝 基吡啶进行计算研究发现, 2,4,6-三氨基-3,5-二硝基吡啶 为一种钝感炸药, 3,5-二氨基-2,4,6-三硝基吡啶的撞击感 度略高于 1,3-二氨基-2,4,6-三硝基苯(DATB), 是有潜在 使用价值的目标炸药. 目前, 国内外广泛研究的多硝基 吡啶类含能化合物主要有: 多硝基吡啶 $(2,4,6$-三硝基吡 啶，TNPy)及其叠氮基衍生物 ${ }^{[22,23]}$, 多氨基多硝基吡啶 (2,6-二氨基-3,5-二硝基吡啶, ANPy 以及 2,4,6-三氨基3,5-二硝基吡啶, TANPy 等 ${ }^{[24,25]}$, 苦基硝基吡啶(2,6-二 苦胺基-3,5-二硝基吡啶, PYX 等 ${ }^{[26,27]}$ ).

\section{1 多硝基/叠氮基吡啶}

由于吡啶环上存在电负性较大的氮原子, 使得环上 氮原子邻、对位的电子云密度减低, 因此, 难以通过直 接硝化过程在吡啶环上引入两个及两个以上硝基. 为了 制得多硝基/叠氮基吡啶类含能化合物常用间接合成方 法, 如万道正 ${ }^{[28]}$ 用 2,2-二硝基乙醇钾(1)在硝酸中经一步 环合反应首次合成出一种稳定的、具有良好爆轰性能的 高效炸药 2,4,6-三硝基吡啶(TNPy). Ritter 和 Licht ${ }^{[29]}$ 也曾 以 2,2-二硝基乙醇钾(1)为原料, 以浓度为 $2 \mathrm{~mol} / \mathrm{L}$ 的磷 酸为作溶剂, 经酸解、环合反应制得一种已知化合物 2,4,6-三硝基吡啶- $N$-氧化物(2, TNPyO), 然后将其在酸 性介质中与亚硝酸钠作用, 脱氧还原得到 2,4,6-三硝基 吡啶(3, TNPy) (Scheme 1).

高氮化合物分子中有较多的 $\mathrm{N}-\mathrm{N}$ 或者 $\mathrm{N}=\mathrm{N}$ 键而 拥有较高的生成热, 使得它们成为潜在的高能材料; 在 化合物分子骨架中引入 1 个或者多个叠氮基是获得超高 氮含能化合物的方法之一[30 34]，每一个叠氮基团可给 化合物增加约 $364 \mathrm{~kJ} / \mathrm{mol}$ 的生成焓 ${ }^{[35]}$. Ritter 和 Licht ${ }^{[29]}$ 发现在 TNPyO 中被活化的 2-,6-硝基可被亲核试剂叠氮 负离子置换, 生成一种高感度炸药 2,6-二叠氮基-4-硝基 吡啶- $N$-氧化物 $(4, \mathrm{DAzPyO})$. Lowe-Ma 等 ${ }^{[36]}$ 研究以廉价 易得的 2,6-二氯吡啶为原料, 经硝化、叠氮化反应可制

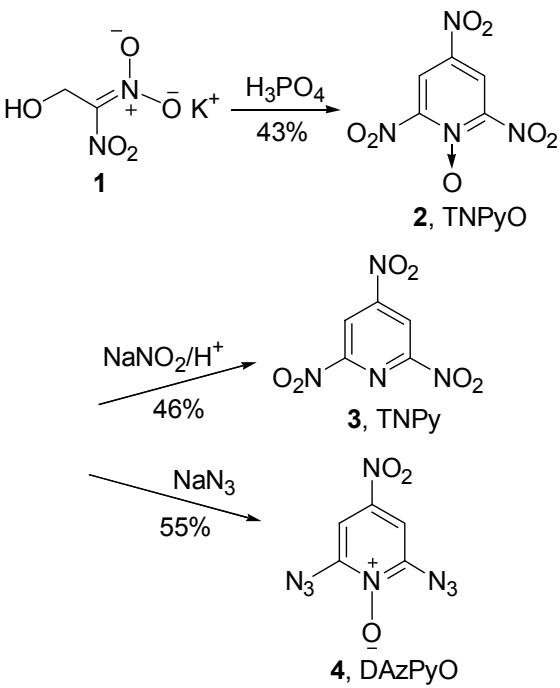

Scheme 1

得 5-叠氮基-6-硝基四唑基 $[1,5-a$ 吡啶(7). 然而化合物 7 极不稳定，在室温下即可脱氮闭环形成 $[1,2,5]$ 啞二唑并 $[3,4-e]$ 四唑并 $[1,5-a]$ 吡啶-3-氧化物 $(8)(\text { Scheme 2) })^{[37]}$.
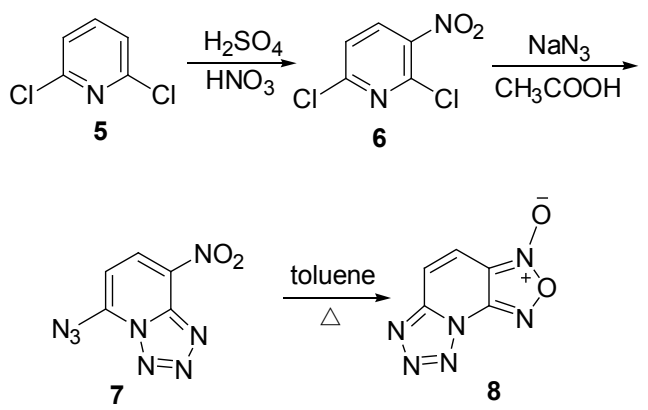

\section{Scheme 2}

由于二苯并四氮杂戊搭烯结构具有较高的热稳定 性, Carboni 等 ${ }^{[38,39]}$ 合成出一类苯系的耐热炸药：四硝基 二苯并-1,3a,4,6 $a$-四氮杂戊搭烯 $(9, \mathrm{TACOT})$, 差热分析 显示该化合物加热至 $354{ }^{\circ} \mathrm{C}$ 以上才会发生轻微的放热 反应. 由于硝基在苯环位置的不同(邻位、间位、对位), 使得 TACOT 有多种异构体，但它们有着相似的爆炸性 能和热动力学性质. 之后, Trudell 等 ${ }^{[40,41]}$ 合成出与 TACOT 结构相近的二吡啶并四氮杂戊搭烯系列含能材 料 14, 18 和 21 (Schemes 3, 4). 他们以不同的三唑并吡 啶 $(11,15)$ 为原料, 在 DMSO 中用碳酸钠作缚酸剂促进 完成 $N$-芳基化反应，生成 $N$-芳基取代的三唑并吡啶 $\mathbf{1 2}$, 16 和 19, 分别经亚磷酸三乙酯还原环化得到内盐 13,17 和 20 , 最后以浓硫酸和浓硝酸硝化得到目标化合物. 差 热分析结果显示 14 的分解峰为 $340 \sim 342{ }^{\circ} \mathrm{C}$, 而 18 的 分解峰为 $396{ }^{\circ} \mathrm{C}$, 晶体密度皆为 $1.88 \mathrm{~g} / \mathrm{cm}^{3}$, 热稳定性 好, 能量优于 TACOT, 而撞击感度低于季戊四醇四硝酸 酯(PETN)和环三亚甲基三硝胺(RDX)，可见，二吡啶并 
四氮杂戊搭烯系列化合物是一类较佳的耐热炸药候选 化合物。

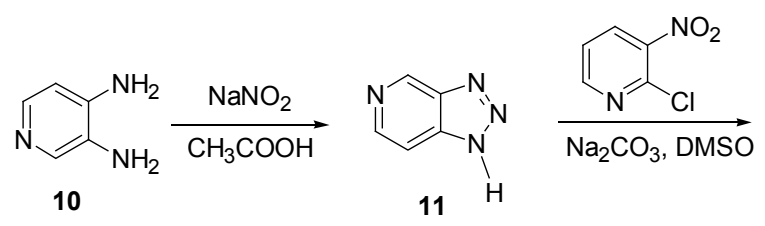<smiles>CCOc1cccnc1-n1nnc2cnccc21</smiles>

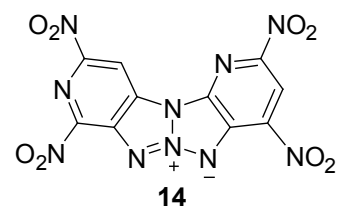<smiles></smiles>

9, TACOT

Scheme 3

与碳环多硝基含能化合物, 如 1,3,5-三硝基苯 (TNB)，2,4,6-三硝基甲苯(TNT)等的合成相比，吡啶环 在结构上的缺电子性使得多硝基/叠氮基吡啶的合成困 难重重. 然而, 2,4,6-三硝基吡啶(3)及 2,4,6-三硝基吡啶$N$-氧化物(2)较之 $\mathrm{TNT}, \mathrm{TNB}$ 却有着更好的密度、氧平衡、 爆速和热稳定性，但撞击感度较高；2,6-二叠氮基-4-硝 基吡啶- $N$-氧化物(4)虽有着爆速高的特点, 但热稳定性 和感度皆不佳(表 1). 大部分高氮多叠氮基化合物高感 度的特点限制了它们大规模应用的可能性.

\section{2 多氨基多硝基吡啶}

在吡啶环系统中交替的引入氨基和硝基基团, 或者 通过对氨基吡啶的硝化形成伯/仲硝胺吡啶可以降低化 合物感度, 提高化合物的稳定性. 为了进一步提高分子 的密度和热稳定性可以将叔胺氧化成相应的 $N$-氧化物 基团, $N$-氧化物中的氮氧键具有双键的特性, 改变了杂 环的电子云分布, 提高了杂环系统的芳香性, 且 $N$-氧化 物基团还可以增加物质的氧平衡, 有利于形成更优的晶 体堆积 ${ }^{[42]}$. 因此，含硝基、氨基、 $N$-氧化物取代的吡啶

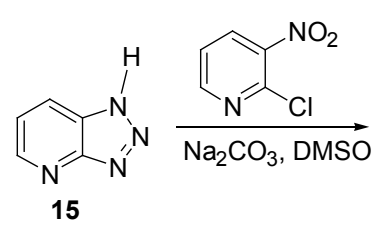<smiles>CCOc1cccnc1-n1nnc2cccnc21</smiles>

16<smiles>O=S(=O)(O)Oc1cccnc1-n1nnc2cccnc21</smiles>

17<smiles></smiles>

18<smiles>CO[Mg]O[Na]</smiles>

15<smiles>CCOC1=CC=C[N+]=C1n1nnc2ncccc21</smiles>

19<smiles>[Z17][n+]1nc2ncc([N+](=O)[O-])cc2n2c3nc([N+](=O)[O-])cc([N+](=O)[O-])c3n21</smiles>

Scheme 4

化合物可作为钝感含能材料如三硝基三氨基苯(TATB), 3-硝基-1,2,4-三唑-5-醖(NTO)等的替代物.

Ritter 和 $\mathrm{Licht}^{[43]}$ 以 2,6-二氨基吡啶(22)为原料，经 混酸硝化和氮氧化反应得到 2,6-二氨基-3,5-二硝基吡 啶- $N$-氧化物(24, ANPyO)，总收率在 $45 \%$ 左右(Scheme $5)$. 该合成路线中第一步的硝化反应伴随有中间体和产 物的酸解、水解副反应，同时硝酸对原料 2,6-二氨基吡 啶的氧化副反应也较明显，需经分离纯化才能得到纯产 品; 另外, 2,6-二氨基-3,5-二硝基吡啶(23)的氮氧化反应 较困难, 终产物中依旧含有较多环氮原子未被氧化的原 料. 为此, 成健等 ${ }^{[44]}$ 对 Ritter-Licht 法进行改进(Scheme $5)$, 通过由发烟硫酸与发烟硝酸或 $\mathrm{KNO}_{3}$ 组成的超酸体 系对 2,6-二氨基吡啶(22)进行硝化，使得化合物 23 的收 率提高至 $90 \%$ 以上，无水的超酸体系有效避免了硝化中 间体以及产物的酸解和硝解; 在 $\mathrm{H}_{2} \mathrm{SO}_{4} / \mathrm{NaHSO}_{4}$ 催化作 用下，对 ANPy 的氮氧化反应相对缓和，反应完全，产 物纯度 $>99 \%$, 但是该改进方法使用超酸硝化，对设备

表 1 一些多硝基/叠氮基吡啶与 TNT, TNB 的部分性能比较 ${ }^{a}$

Table 1 Properties of some polynitro/azidopyridines and their comparison with TNT, TNB

\begin{tabular}{lcclcccc}
\hline Compd. & $\rho /\left({\left.\mathrm{g} \cdot \mathrm{cm}^{-3}\right)}^{-3}\right.$ & O.B. $/ \%$ & m.p. $/{ }^{\circ} \mathrm{C}$ & $Q /\left(\mathrm{kJ}^{\circ} \mathrm{mol}^{-1}\right)$ & $D /\left(\mathrm{m}^{-1}{ }^{-1}\right)$ & I.S./J & F.S./N \\
\hline TNPy (3) & 1.77 & -37.4 & 162 & 893 & 8161 & $4.5 \sim 6.5$ & $>36$ \\
TNPyO (2) & 1.86 & -27.8 & $170(\mathrm{dec})$. & 1217 & 8369 & $1.5 \sim 3.0$ & 16 \\
DazPyO (4) & - & -57.6 & $100(\mathrm{dec})$. & - & 8532 & $<1.5$ & 10 \\
TNT & 1.64 & -73.9 & 81.8 & 1026 & 6666 & 15 & $>36$ \\
TNB & 1.68 & -56.3 & 123 & 1043 & 7277 & 7.5 & $>36$ \\
\hline
\end{tabular}

${ }^{a} \rho$, density; $Q$, Heat of explosion; $D$, detonation velocity; I.S., impact sensitivity; F.S., friction sensitivity. 
要求较高和工艺过程较复杂而难于工业化.<smiles>Nc1cccc(N)n1</smiles>

22

23, ANPy

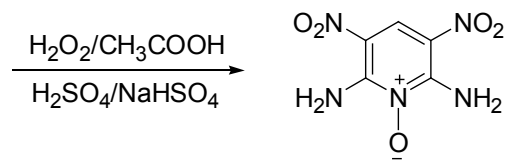

24, ANPyO

Scheme 5

考虑到吡啶环上若存在多个硝基基团会降低氮原 子的氧化活性, 而多个氨基的存在在强烈的硝化条件下 也易发生氧化副反应，成健等 ${ }^{[45]}$ 提出以 2,6 -二氨基吡啶 为原料, 经乙酰化、氮氧化和硝化三步反应得到 ANPyO 的新方法(Scheme 6). 该法利用 2,6-二乙酰氨基吡定环 氮原子的高氧化活性, 在室温无催化剂条件下完成氮氧 化反应; 在 2,6-二乙酰氨基吡定-1-氧化物(25, DAPO)中 受到保护的氨基在硝化体系中几乎无氧化副反应发生, 分子中存在的 $\mathrm{N}^{+} \rightarrow \mathrm{O}^{-}$结构使得吡啶环 $\pi$ 电子云密度增 加, 同时也避免了环氮原子在酸性体系中的质子化, 使 2,6-二乙酰氨基吡啶-1-氧化物(25)较易在低温下完成硝 化反应. 该法中先氮氧化再硝化的改进思路尽管可以提 高反应物的硝化反应能力, 但同时不可避免地会改变硝 基的取代位置, 产生吡啶环 4-位被硝化的副反应而降低 目标产物收率, 并且硝化过程依旧需采用发烟硫酸/发 烟硝酸的强硝化体系. 成健等 ${ }^{[46]}$ 还尝试使用 $N, N, N$-三甲 基- $N$-丙磺酸基-硫酸氢铵 $\left(\mathrm{TMPSHSO}_{4}\right.$ ) 为催化剂, 以硝 基甲烷为溶剂, $\mathrm{N}_{2} \mathrm{O}_{5}$ 为硝化剂硝化 2,6-二乙酰氨基吡啶1-氧化物(25)合成 ANPyO.

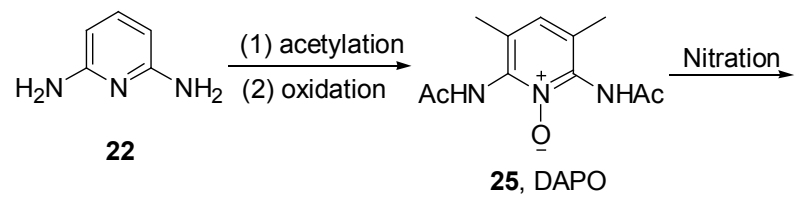<smiles></smiles>

Scheme 6

王艳红等 ${ }^{\left[{ }^{[47}\right]}$ 先将 2, 6-二氨基吡啶(22)制成易保存的 硫酸盐(26), 然后在室温条件下也以发烟硫酸和发烟硝 酸作为硝化剂硝化得到 ANPy, 进一步室温氧化得到

ANPyO (Scheme 7). 姚其正等 ${ }^{[48]}$ 以价格低廉、易得的 2,6-二氯吡啶为原料, 经浓硫酸/硝酸钾体系和 $\mathrm{NH}_{3} / \mathrm{C}_{2} \mathrm{H}_{5} \mathrm{OH}$ 进行交替的硝化、氨化过程即可得到 ANPy (Scheme 8). 该法无需采用强硝化体系，后处理过程简 单, 产品纯度高, 基本不需要进行其他纯化处理即可用 于下一步反应，但是该法反应步骤较长，有碍于其在工 业生产中的应用.

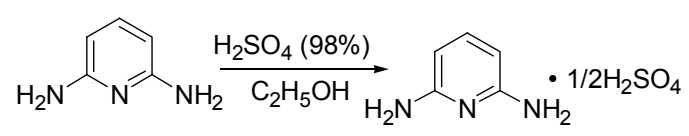

22

26<smiles>Nc1nc(N)c([N+](=O)[O-])cc1[N+](=O)[O-]</smiles>

24, ANPyO

Scheme 7
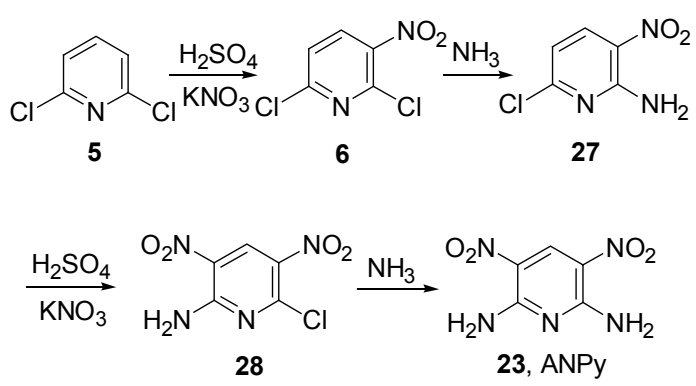

Scheme 8

鉴于 ANPyO 的结构与 TATB 相似, 其合成成本显 著低于 TATB, 在含能领域具有广阔的应用前景 ${ }^{[45,49]}$, 刘祖亮课题组重点从溶剂精制、机械混合、结晶包覆、 添加高聚物黏结剂等方面对含有 ANPyO 炸药的性能进 行了应用研究. 何志伟等 ${ }^{[50,51]}$ 发现用 $\mathrm{CF}_{3} \mathrm{COOH}$ 精制的 ANPyO 机械感度和摩擦感度低, 采用结晶包覆的方法 制备 $\mathrm{ANPyO} / \mathrm{RDX}$ 的复合物撞击感度和摩擦感度较 $\mathrm{RDX}$ 均有显著下降, 对单质 $\mathrm{ANPyO}$ 进行适当的表面改 性或包覆能提高其综合性能 ${ }^{[52 \sim 56]}$.

VNS 氧化胺化法是一种以 $\mathrm{X}-\mathrm{NH}_{2}$ (常用羟胺, 即 $\mathrm{X}=\mathrm{OH}$ ) 为胺化剂, $\mathrm{KMnO}_{4}$ 或 $\mathrm{H}_{2} \mathrm{O}_{2}$ 等作为氧化剂, 在低 温(如一 $33{ }^{\circ} \mathrm{C}$ ) 条件下实现硝基芳烃的胺化反应的方 法 ${ }^{[57 ~ 60]}$. Woźniak 和 van der Plas 等 ${ }^{[61,62]}$ 系统研究过硝基 吡啶的氧化胺化反应，并成功合成出 2,4,6-三氨基-3,5二硝基吡啶(29, TANPy), 其晶体密度为 $1.819 \mathrm{~g} / \mathrm{cm}^{3}$, 分 解点为 $353{ }^{\circ} \mathrm{C}$. Hollins 等 ${ }^{[63]}$ 先用此法合成出 TANPy, 再 将其进一步氧化得到一种全取代的吡啶类钝感含能化 合物 2,4,6-三氨基-3,5-二硝基吡啶-1-氧化物(30, TAN$\mathrm{PyO})$, 其晶体密度为 $1.876 \mathrm{~g} / \mathrm{cm}^{3}$, 分解点为 $308{ }^{\circ} \mathrm{C}$; 化 合物 30 亦可直接用 $\mathrm{ANPyO}$ 进行 VNS 胺化得到(Scheme 
9). 与 ANPyO 的合成相比, 因要在吡啶环 4-位引入氨基 使得 TANPyO 的合成步骤较长, 方法较复杂, 其中使用 的氧化胺化法虽然反应的主要副产物是水, 对环境无 害, 但该法存在反应条件苛刻 $\left(-33{ }^{\circ} \mathrm{C}\right)$ 和选择性差等 缺陷, 并因 TANPyO 的性能未明显优于 ANPyO, 应用 价值不高, 使其合成主要停留在理论研究方面.<smiles>Nc1nc(N)c([N+](=O)[O-])cc1[N+](=O)[O-]</smiles>

23, ANPy<smiles>Nc1c([N+](=O)[O-])c(N)[n+]([O-])c(N)c1[N+](=O)[O-]</smiles>

30, TANPyO

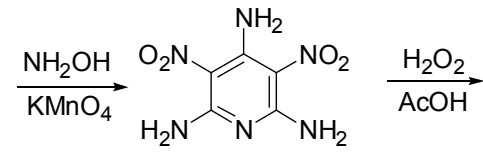

29, TANPy<smiles>Nc1c([N+](=O)[O-])cc([N+](=O)[O-])c(N)[n+]1[O-]</smiles>

24, ANPyO

\section{Scheme 9}

硝胺是一类高能量密度材料, 主要代表性化合物有 1,3,5-三硝基-1,3,5-三氮杂环己烷(RDX)和 1,3,5,7-四硝 基-1,3,5,7-四氮杂环辛烷(HMX) ${ }^{[64]}$. 通过吡啶环上氨/胺 基的硝化可得到伯硝胺或仲硝胺，伯硝胺吡啶化合物可 发生重排生成邻氨基硝基化合物, 例如化合物 $\mathbf{4 0}$ 的合 成即以 4-氨基-2-氯吡啶为原料, 经硝化所得中间体伯 硝胺重排而得; 但仲硝胺却是稳定的炸药. Ritter 和 Lich $\mathrm{t}^{\left[{ }^{[5]}\right.}$ 以 3,5-二硝基吡啶衍生物为原料, 通过甲胺取 代、硝化两步反应合成出多种仲硝胺取代的吡啶类含能 化合物 (Scheme 10). 姚其正等 ${ }^{\left[{ }^{[6]}\right.}$ 以 4-氨基-2-氯吡啶为 原料，通过硝化、亲核取代反应得到一系列 4-氨基-3,5-

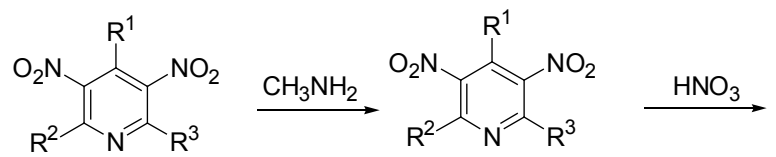

31: $R^{1}=H, R^{2}=N_{2}, R^{3}=N_{2}$ 32: $R^{1}=H, R^{2}=N_{C H}, R^{3}=N_{3 C H}$

34: $\mathrm{R}^{1}=\mathrm{H}, \mathrm{R}^{2}=\mathrm{H}, \mathrm{R}^{3}=\mathrm{Cl} \quad 35: \mathrm{R}^{1}=\mathrm{H}, \mathrm{R}^{2}=\mathrm{H}, \mathrm{R}^{3}=\mathrm{NHCH}_{3}$

37: $R^{1}=N_{2}, R^{2}=H, R^{3}=H \quad 38: R^{1}=N_{H C H}, R^{2}=H, R^{3}=H$<smiles>[R]c1nc([R])c([N+](=O)[O-])c([R])c1[N+](=O)[O-]</smiles>

33: $\mathrm{R}^{1}=\mathrm{H}, \mathrm{R}^{2}=\mathrm{O}_{2} \mathrm{NNCH}_{3}, \mathrm{R}^{3}=\mathrm{O}_{2} \mathrm{NNCH}_{3}$

36: $\mathrm{R}^{1}=\mathrm{H}, \mathrm{R}^{2}=\mathrm{H}, \mathrm{R}^{3}=\mathrm{O}_{2} \mathrm{NNCH}_{3}$

39: $\mathrm{R}^{1}=\mathrm{O}_{2} \mathrm{NNCH}_{3}, \mathrm{R}^{2}=\mathrm{H}, \mathrm{R}_{3}=\mathrm{H}$

\section{Scheme 10}

二硝基吡啶衍生物(Scheme 11).

\section{3 苦基硝基吡啶}

炸药的安定性是指在一定条件下，炸药保持其物 理、化学及爆炸性质不发生可觉察的变化或发生在允许 范围内变化的能力, 是评价炸药性能优劣, 甚至是有无 应用价值的主要依据. 炸药合成的实践表明, 较多能量 特性优良的炸药往往安定性不佳，如 RDX 酩 ${ }^{[67,68]}$ 、四硝 基甘脲 ${ }^{[69,70]}$ 等. 为了满足宇宙航行、深井作业等方面的 需要, 研发耐热炸药受到国内外炸药界的极大关注. 这 类炸药热安定性较高, 具有较高熔点和较低的蒸汽压, 经长期加热和冷却后仍能可靠地起爆, 同时具有适宜的 撞击感度和较高的能量.

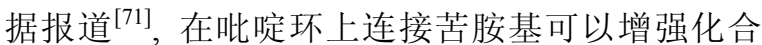
物的热稳定性等性能. 美国 Los Alamos 国家实验室曾设

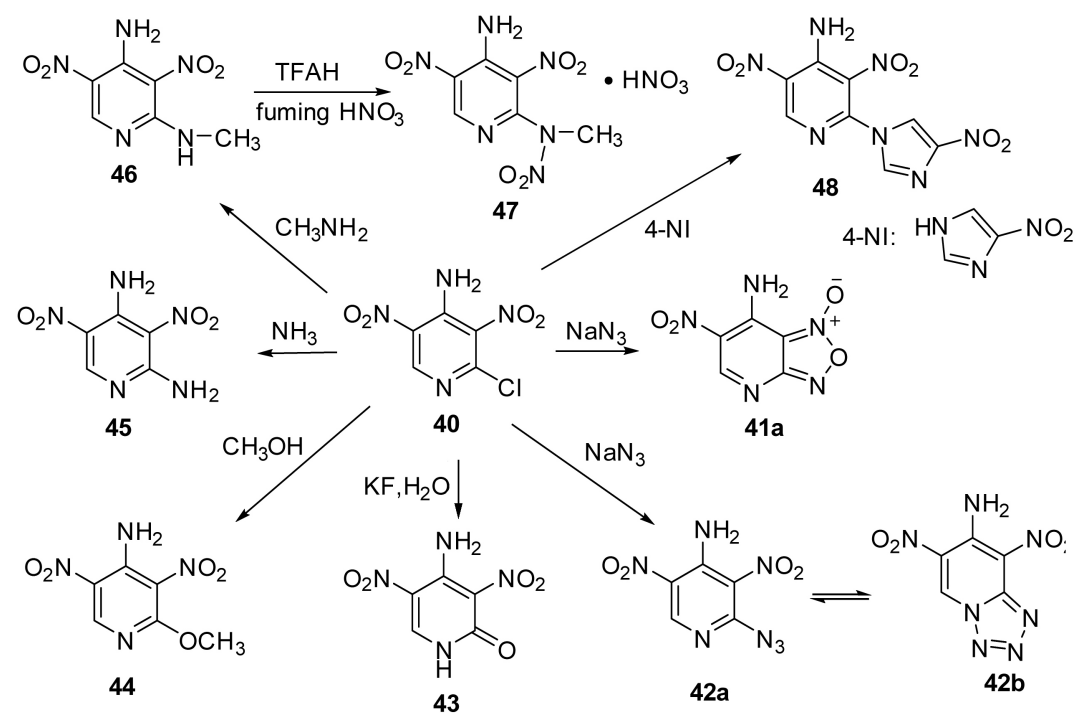

Scheme 11 
计、合成出苦基取代的吡啶环系耐热含能材料 2,6-双(苦 胺基)-3,5-二硝基吡啶(51, PYX) (Scheme 12). 它是美国 原子能委员会研究和发展的一种中等威力的耐热炸药, 比任何其他氧平衡相当的已知炸药的热安定性更好. 同 时, 它是化学惰性的, 在高温下无蒸汽压力 ${ }^{[72,73]}$. PYX 分子的稳定性很大程度上取决于吡啶环的贡献, 处于 PYX 分子中央的吡啶环内氮杂原子采用 $\mathrm{sp}^{2}$ 杂化方式, 其中一个杂化轨道为非键轨道保留有一对孤电子, 另两 个杂化轨道与相邻碳原子形成 $\sigma$ 键骨架, 未参与杂化的 单电子 $\mathrm{Z}$ 轨道与 5 个碳原子的 $\mathrm{P}_{\mathrm{Z}}$ 轨道形成 $\pi_{6}^{6}$ 共轭体系. 且吡啶环内氮原子具有类似硝基苯的针化作用, 能引起 整个分子 $\pi$ 体系环流的微扰改变, 结果使共轭在整个分 子中重新调整最后趋于均衡, 使得分子内原子间作用力 更加强烈, 以致分子的位能获得了因氮原子引起的额外 的 $\pi$ 能降 ${ }^{[74]}$.<smiles>O=[N+]([O-])c1cc([N+](=O)[O-])c(Cl)c([N+](=O)[O-])c1</smiles>

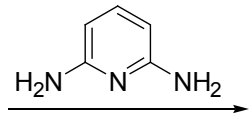

49<smiles>O=[N+]([O-])c1cc([N+](=O)[O-])c(Nc2cccc(Nc3c([N+](=O)[O-])cc([N+](=O)[O-])cc3[N+](=O)[O-])n2)c([N+](=O)[O-])c1</smiles><smiles>O=[N+]([O-])c1cc([N+](=O)[O-])c(Nc2nc(Nc3c([N+](=O)[O-])cc([N+](=O)[O-])cc3[N+](=O)[O-])c([N+](=O)[O-])cc2[N+](=O)[O-])c([N+](=O)[O-])c1</smiles>

51, PYX

\section{Scheme 12}

由于生产 PYX 所需要的主要原料 2,6-二氨基吡啶 和苦基氯价格较贵, 从而影响了 PYX 的实际应用. 王乃 兴等 ${ }^{[75]}$ 以价格低廉、来源广泛的 2-氨基吡啶(52)和 2,4二硝基氯苯(53)为原料经缩合和硝化两步反应合成出 2苦胺基-3,5-二硝基吡啶(55), 其分解点为 $268{ }^{\circ} \mathrm{C}$, 密度 为 $1.83 \mathrm{~g} / \mathrm{cm}^{3}$ (Scheme 13). 之后, 王乃兴等 ${ }^{[76]}$ 又用 52 和 1,3,5-三氯-2,4,6-三硝基苯(56)经三步反应得到 $N$-(2,4,6三硝基-3,5-二氨基苯基)-3,5-二硝基-2-氨基吡啶(59), 其 分解点为 $280.5{ }^{\circ} \mathrm{C}$, 晶体密度为 $1.91 \mathrm{~g} / \mathrm{cm}^{3}$ (Scheme 14).

虽然化合物 55 和 59 仅仅为卤代多硝基苯与 2 -氨基 吡啶的缩合衍生物, 但其成功合成为 PYX 在国内的合 成工艺改进和创新提供了依据, 王乃兴等 ${ }^{[77,78]}$ 利用卤代 二硝基苯和 2,6-二氨基吡啶进行缩合和硝化反应合成出<smiles>Nc1ccccn1</smiles>

52

53

54<smiles>O=[N+]([O-])c1cc([N+](=O)[O-])c(Nc2ncc(N([O-])O[Na])cc2[N+](=O)[O-])c([N+](=O)[O-])c1</smiles>

55

Scheme 13<smiles>Cc1ccc(Nc2c(Nc3c([N+](=O)[O-])c(Cl)c([N+](=O)[O-])c(Cl)c3[N+](=O)[O-])c([N+](=O)[O-])c(Cl)c(Cl)c2[N+](=O)[O-])nc1</smiles><smiles>O=[N+]([O-])c1cc([N+](=O)[O-])c(Nc2ncc([N+](=O)[O-])c(Cl)c2[N+](=O)[O-])c([N+](=O)[O-])c1</smiles><smiles>Nc1c([N+](=O)[O-])c(N)c([N+](=O)[O-])c(Nc2ncc([N+](=O)[O-])cc2[N+](=O)[O-])c1[N+](=O)[O-]</smiles>

59

Scheme 14

2,6-二苦胺基-3,5-二硝基吡啶(51, PYX) (Scheme 15), 该 法有效避免了 49 在碱性条件下水解生成苦味酸, 终止 与 2, 6-二氨基吡啶进行缩合, 并且容易引起爆炸的缺 点. 王乃兴等 ${ }^{[79]}$ 尝试使用 $1,3,5$-三氯-2,4,6-三硝基苯(56) 和 2,6-二氨基吡啶(22)反应, 合成出结构类似于 PYX 的 耐热性能更佳的含能化合物 $N, N^{\prime}$-双(2,4,6-三硝基-3,5二氨基苯基)-3,5-二硝基-2,6-二氨基吡啶(64) (Scheme 16).

Coburn 等 ${ }^{[80]}$ 以多氨基吡啶为原料, 经苦基取代、硝 化得到多种 PYX 的衍生物，包括 2,5-二苦胺基-3,6-二硝 基吡啶(67)、3,5-二苦胺基-2,6-二硝基吡啶(70)、2,4,6三苦胺基-3,5-二硝基吡啶(73) (Scheme 17), 部分苦基硝 基取代的吡啶类含能化合物的性能如表 2 所示 ${ }^{[64,81]}$.

\section{2 吡啶类含能离子盐}

高能量密度材料(HEDMs)的能量来源主要有两种: (1)碳骨架的氧化，代表性化合物有 TNT，RDX，HMX; (2)生成热高, 代表性化合物有 CL-20, TNAZ, FOX-7, 富氮杂环含能离子盐等 ${ }^{[82]}$. 近年来, 由于富氮杂环含能 
<smiles>[R]c1ccc([N+](=O)[O-])cc1[N+](=O)[O-]</smiles>

60: $\mathrm{R}^{1}=\mathrm{F} ; 53: \mathrm{R}^{1}=\mathrm{Cl}$<smiles>O=[N+]([O-])c1ccc(Nc2cccc(Nc3ccc([N+](=O)[O-])cc3[N+](=O)[O-])n2)c([N+](=O)[O-])c1</smiles><smiles>O=[N+]([O-])c1cc([N+](=O)[O-])c(Nc2nc(Nc3c([N+](=O)[O-])cc([N+](=O)[O-])cc3[N+](=O)[O-])c([N+](=O)[O-])cc2[N+](=O)[O-])c([N+](=O)[O-])c1</smiles>

51, PYX

Scheme 15<smiles>Nc1cccc(N)n1</smiles><smiles>O=[N+]([O-])c1c(Cl)c([N+](=O)[O-])c(Nc2cccc(Nc3c([N+](=O)[O-])c(Cl)c([N+](=O)[O-])c([N+](=O)[O-])c3[N+](=O)[O-])n2)c([N+](=O)[O-])c1Cl</smiles><smiles>O=[N+]([O-])c1cc([N+](=O)[O-])c(Nc2nc(Nc3c([N+](=O)[O-])c(Cl)c([N+](=O)[O-])c(Cl)c3[N+](=O)[O-])c([N+](=O)[O-])c([N+](=O)[O-])c2[N+](=O)[O-])c([N+](=O)[O-])c1Cl</smiles><smiles>Nc1c([N+](=O)[O-])c(N)c([N+](=O)[O-])c(Nc2nc(Nc3c([N+](=O)[O-])c(N)c([N+](=O)[O-])c([N+](=O)[O-])c3[N+](=O)[O-])c([N+](=O)[O-])cc2[N+](=O)[O-])c1[N+](=O)[O-]</smiles>

\section{Scheme 16}

离子盐的分子结构中含有大量的 $\mathrm{N}-\mathrm{N}$ 键和 $\mathrm{C}-\mathrm{N}$ 键, 氮元素百分含量高, 致使其主要分解产物为氮气, 对环 境友好; 含能离子盐的阴阳离子可修饰程度高, 引起较 多含能材料研究者的兴趣 ${ }^{[83 ~ 92]}$. 虽然吡啶类含能化合

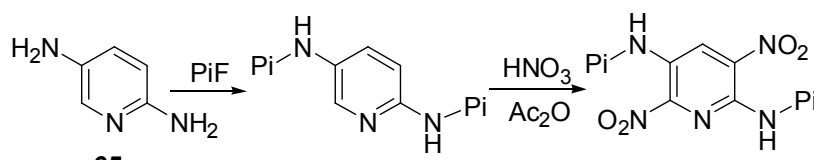

67<smiles>Nc1cncc(N)c1</smiles>

68

69<smiles>CCCNc1cc(NCCC)c([N+](=O)[O-])nc1[N+](=O)[O-]</smiles>

70

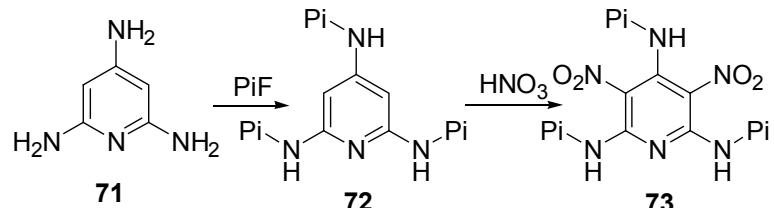<smiles>Cc1c([N+](=O)[O-])cc([N+](=O)[O-])cc1[N+](=O)[O-]</smiles>

Scheme 17

表 2 部分苦基硝基取代的吡啶类含能化合物的性能 ${ }^{a}$

Table 2 Some explosive properties of picryl- and nitro-substituted energetic pyridines

\begin{tabular}{|c|c|c|c|c|c|c|}
\hline Compd. & $\begin{array}{c}\rho / \\
\left(\mathrm{g} \bullet \mathrm{cm}^{-3}\right)\end{array}$ & $\begin{array}{c}\text { m.p./ } \\
{ }^{\circ} \mathrm{C}\end{array}$ & $\begin{array}{c}\text { DTA/ } \\
{ }^{\circ} \mathrm{C}\end{array}$ & $\begin{array}{l}\text { C-J pres- } \\
\text { sure/GPa }\end{array}$ & $\begin{array}{c}D / \\
\left(\mathrm{km} \cdot \mathrm{s}^{-1}\right)\end{array}$ & I.S. $/ \mathrm{cm}$ \\
\hline 70 & 1.77 & $\begin{array}{c}311 \\
\text { (dec.) }\end{array}$ & 300 & 24. 2 & 7.45 & $4.5 \sim 6.5$ \\
\hline 51 & 1.75 & 460 & 350 & 24.2 & 7. 45 & 15 \\
\hline 73 & 1. 80 & $\begin{array}{c}276 \\
\text { (dec.) }\end{array}$ & 260 & 26.3 & 7. 86 & 7.5 \\
\hline
\end{tabular}

${ }^{a} \rho$, Crystal density; DTA, Thermal stability; C-J pressure, Calculated values; $D$, detonation velocity; I.S., impact sensitivity.

物具有相对良好的热稳定性和性能特点, 但因吡啶环中 的氮元素含量偏低，故被选择作吡啶含能离子盐的前体 化合物较少，仅有 2-羟基-3,5-二硝基吡啶(2HDNP)，4着基-3,5-二硝基吡啶(4HDNP)，4-着基-3.5-二硝基吡啶 氮氧化物(4HDNPO)等 ${ }^{[93,94]}$.

郑玉梅等 ${ }^{[95]}$ 合成出 4-差基-3,5-二硝基吡啶铜盐 (74)、4-羊基-3,5-二硝基吡啶铅盐(75)、2-羊基-3,5-二硝 基吡啶铜盐(76)、2-羊基-3,5-二硝基吡啶铅盐(77)、4-看 基-3,5-二硝基吡啶氮氧化物的铜盐(78)和 4-羊基-3,5-二 硝基吡啶氮氧化物的铅盐(79) (Scheme 18). 赵风起 等 ${ }^{[95 ~ 97]}$ 在此基础上对化合物 74 79 进行性能研究发现 77 比 75 有着更好的耐热性能、催化燃烧和降低压力指 
数的作用; 78 和 79 对 $\mathrm{RDX}, \mathrm{AP}, \mathrm{NC}+\mathrm{NG}$ 混合物的热分 解有较明显的催化作用, 且在催化热分解时放出比纯 $\mathrm{RDX}$ 及 $\mathrm{NC}+\mathrm{NG}$ 等较大的分解热; 74,76 和 78 低压下使 RDX-CMDB 推进剂燃速稍有提高, 中高压下的燃速却 略有降低; 75, 77 和 79 皆有较好的催化燃烧作用和降低 压力指数的能力, 其中 $\mathbf{7 7}$ 的催化效率最高, 降低压力指 数能力最强.

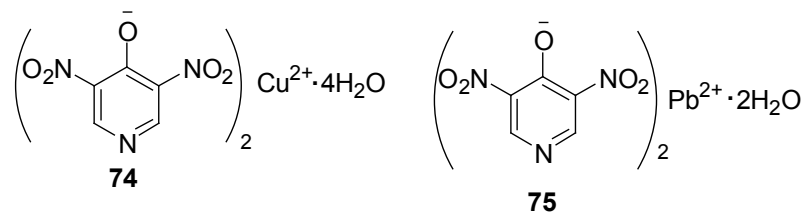

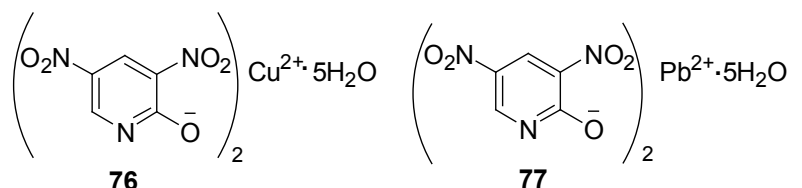

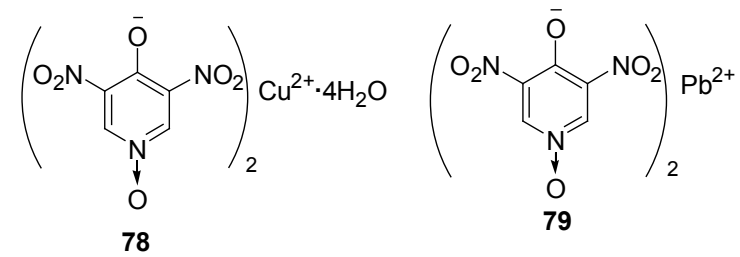

Scheme 18
张国防等以多硝基吡啶酮为原料，合成出它们的碱 金属盐 ${ }^{[98]}$ 、碱土金属盐 ${ }^{[99]}$ 、过渡金属盐 ${ }^{[100]}$ 和稀土金属 盐，通过对盐的单晶结构分析发现稀土金属离子和碱金 属离子与硝基上氧原子之间有比较强的作用，而过渡金 属离子主要与吡啶环上的氮原子有比较强的配位作用, 出现该现象的主要原因为硬酸和硬碱之间有更强的亲 和力 ${ }^{[101]}$. 该课题组在此基础上设计、合成出多种吡啶酮 类高氮含能盐, 其中包括 2HDNP, 4HDNP 的铵盐、二乙 胺盐、肼盐、碳酸胍盐和 3-氨基-1,2,4-三氮唑盐, $4 \mathrm{HDNPO}$ 的肼盐、碳酸胍盐 ${ }^{[102]}$. 最近, Muralidharan 等 ${ }^{[103]}$ 以中性的 3,5-二硝基-2-羟基吡啶(DNP)为前体合 成出一系列的吡啶类含能离子盐 $80 \sim 92$ (Scheme 19), 其阳离子主要有艮、胍、氨基胍、硝基胍、1,3-二氨基 胍、3-氨基-1,2,4-三唑、3,5-二氨基-1,2,4-三唑、4-硝基 咪唑、3-硝基-1,2,4-三唑、3,4-二氨基呋咱等, 进一步增 加了 3,5-二硝基吡啶类含能离子盐的结构多样化.

与传统的含能化合物相比, 含能离子盐具有更低的 蒸汽压和更高的密度, 比原子种类和数目相近的类似物 热稳定性更好 ${ }^{[103]}$. 然而, 吡啶类化合物氮元素的百分 含量普遍偏低, 而碳氢元素的含量较高, 影响到该类化 合物在作为含能离子盐阴离子的使用机率，总的研究趋 势可采用以高氮含量的含能化合物作为阳离子来提高 吡啶类含能离子盐的总体性能.

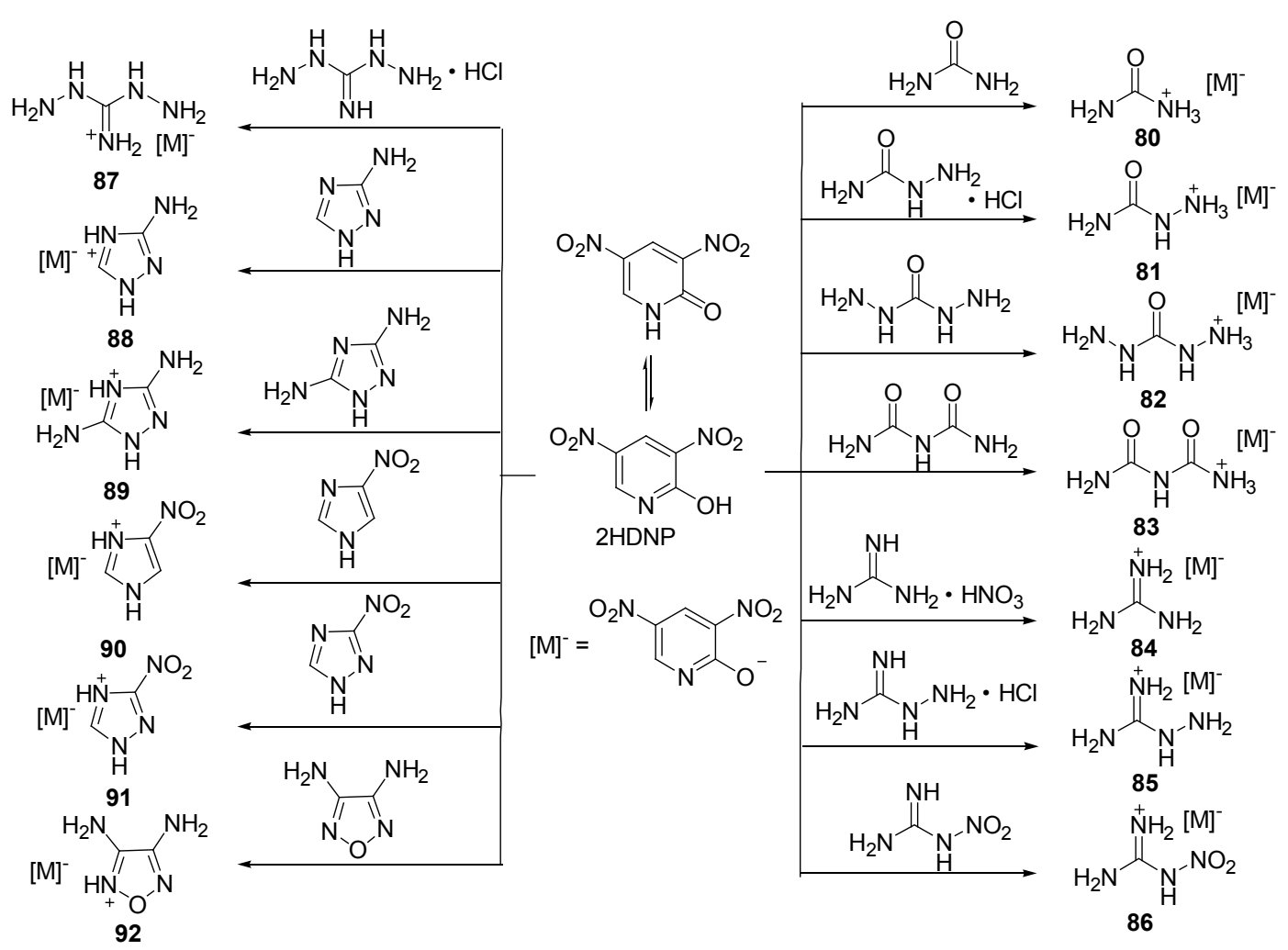

Scheme 19 


\section{3 吡啶类含能配合物}

配合物是由中心金属离子、配体和外界离子相结合 而形成的一类化合物, 而以含能材料为配体形成的、具 有一定爆炸性能的配合物称为含能配合物 ${ }^{[04]}$. 从分子 设计的观点出发, 作为含能配合物的配体必须满足两个 条件: 一是含碳氢量少而含氮量高, 这样才具有高的正 生成焓, 使得生成物能量高, 同时高氮、低碳氢含量不 仅使其具有较高的密度, 而且更容易达到氧平衡; 二是 至少含有一个能提供孤对电子的配位原子, 如 $\mathrm{N}, \mathrm{O}, \mathrm{S}$ 等. 根据上述条件, 多氨基硝基吡啶氮氧化物中的氨基 氮原子及其环氮原子都具有孤对电子, 能与中心离子形 成配位键. 在这类配合物中, 以 ANPyO 及其衍生物作 为配体的配合物具有良好的安定性和催化性能, 从而受 到含能材料研究人员的重视 ${ }^{[105 ~ 107]}$.

ANPyO 分子结构呈平面状, 氨基氢原子与硝基氧 原子、氮氧基氧原子分别形成分子内、分子间氢键, 这 就必将导致晶体呈层状或片状结构. 刘祖亮等 ${ }^{[108 \sim 112]}$ 以 ANPyO 为配体制得多种过渡金属配合物，用 $X$ 射线单 晶衍射法测定分子结构, 它们的部分晶体学参数见表 3 .

由单晶的测试结果可知, ANPyO 的过渡金属配合物 中皆有溶剂分子参与配位, ANPyO 作为双齿配体, 其中 氮氧键中的氧原子以其孤对电子参与过渡金属配位，2位的氨基脱去质子成负离子与中心金属离子配位 ${ }^{[113]}$. 脱去质子的 ANPyO 与金属离子配位形成稳定的五元螯 合环，其中的 5 个原子具有较好的共面性，因而配合物 分子结构稳定, 具体表现为耐热性好、不吸湿、不水解
等 ${ }^{[112]}$.

炸药的感度是指炸药在外界能量作用下发生爆炸 的难易程度，是炸药使用安全性能的一种量度. 刘祖亮 等对以 ANPyO 为配体的过渡金属配合物的感度进行了 部分测试，与 ANPyO 的性能进行对比发现，撞击感度 有所降低, 摩擦感度皆为零 (表 4); 他们还研究了 ANPyO 的过渡金属配合物对高氯酸铵(AP)热分解的催 化作用，发现它们可以加速 AP 的分解，使 AP 的热分解 提前，分解速度加快，具有良好的催化效果(表 4).

目前吡啶类含能配合物的研究主要以 ANPyO 为配 体，集中在配合物的制备与表征方面，其中培养并测试 化合物的晶体结构仍然是一个难点. 然而, 配合物的结 构受到诸多因素的影响，如溶剂、反应条件等，使得很 难对其结构进行预测. 因此可通过改变中心金属离子、 调控配合物分子中配体数目、调控合成过程等方式寻找 性能优良的配合物，以及对已有的配合物结构和性能进 行分析，再有目的地设计和选择合适的配体与金属离子 及其控制反应条件等因素来系统研究配合物的结构与 性能，对指导实验研究具有重要意义.

\section{4 结论与展望}

综上所述，在吡啶环上可引入多个硝基等爆炸性基 才和多种功能性基团，使其爆炸性能得以改变或改善， 形成的吡啶类含能化合物有较高的密度和能量, 特别是 其中部分化合物表现出的高能钝感特性，是一类具有研 究价值和广泛应用前景的含能材料. 从化合物分子结构

表 $3 \mathrm{ANPyO}$ 及其过渡金属配合物的晶体学参数测试结果 ${ }^{[108 ~ 112]}$

Table 3 Crystallographic data of ANPyO and its transition metal complexes

\begin{tabular}{llllllll}
\hline \multicolumn{1}{c}{ Compd. } & Space group & Crystal system & \multicolumn{1}{c}{$a / \mathrm{nm}$} & \multicolumn{1}{c}{$b / \mathrm{nm}$} & $c / \mathrm{nm}$ & $V / \mathrm{nm}^{3}$ & $D_{\mathrm{c}} /\left(\mathrm{g} \bullet \mathrm{cm}^{-3}\right)$ \\
\hline ANPyO & Monoclinic & $C 2 / C$ & $1.4740(3)$ & $0.72920(15)$ & $0.75740(15)$ & $0.7568(3)$ & 1.888 \\
{$\left[\mathrm{~Pb}_{2}\left(\mathrm{C}_{5} \mathrm{H}_{3} \mathrm{~N}_{5} \mathrm{O}_{5}\right)_{2}(\mathrm{NMP}) \cdot \mathrm{NMP}\right]_{n}$} & Tetragonal & $P 43$ & $1.31297(3)$ & $1.31297(3)$ & $1.67858(8)$ & $2.8937(17)$ & 2.385 \\
$\mathrm{Ni}_{(}\left(\mathrm{C}_{5} \mathrm{~N}_{5} \mathrm{O}_{5} \mathrm{H}_{4}\right)_{2}(\mathrm{by})_{2} \cdot 2 \mathrm{by}$ & Monoclinic & $P 21 / C$ & $9.0070(18)$ & $10.002(2)$ & $19.445(4)$ & $1.7481(6)$ & 1.526 \\
$\mathrm{Fe}\left(\mathrm{C}_{5} \mathrm{~N}_{5} \mathrm{O}_{5} \mathrm{H}_{4}\right)_{3} \cdot 3 \mathrm{DMF} \bullet 1 / 2 \mathrm{H}_{2} \mathrm{O}$ & Triclinic & $P 1$ & $1.1715(2)$ & $1.2900(3)$ & $1.4978(3)$ & $2.0104(8)$ & 1.531 \\
$\mathrm{Co}\left(\mathrm{C}_{5} \mathrm{~N}_{5} \mathrm{O}_{5} \mathrm{H}_{4}\right)_{3} \cdot 3 \mathrm{DMF}$ & Triclinic & $P 1$ & $1.1808(2)$ & $1.2955(3)$ & $1.4764(3)$ & $1.8924(10)$ & 1.616 \\
$\mathrm{Cu}_{4} \mathrm{O}\left(\mathrm{C}_{5} \mathrm{~N}_{5} \mathrm{O}_{5} \mathrm{H}_{3}\right)_{2}\left(\mathrm{CH}_{3} \mathrm{COO}\right)_{2-}-$ & Triclinic & $P 1$ & $8.6710(17)$ & $11.226(2)$ & $18.741(4)$ & $1.6351(6)$ & 1.957 \\
$\left(\mathrm{CH}_{3} \mathrm{O}\right)_{2} \cdot \mathrm{DMF}$ & & & & & &
\end{tabular}

表 4 ANPyO 及其过渡金属配合物的感度性能对比 ${ }^{[107 ~ 112] ~}$

Table 4 Sensitivity performances cont rasting of ANPyO and its transition metal complexes

\begin{tabular}{lcccc}
\hline \multicolumn{1}{c}{ Compd. } & Impact sensitivity/cm & Friction sensitivity/\% & Shock sensitivity/mm & Thermal decomposition of AP/ ${ }^{\circ} \mathrm{C}$ \\
\hline $\mathrm{ANPyO}$ & 250 & 0 & 5.6 & - \\
{$\left[\mathrm{Pb}_{2}\left(\mathrm{C}_{5} \mathrm{H}_{3} \mathrm{~N}_{5} \mathrm{O}_{5}\right)_{2}(\mathrm{NMP}) \cdot \mathrm{NMP}\right]_{n}$} & - & - & - & $54.42($ lower $)$ \\
$\mathrm{Ni}_{2}\left(\mathrm{C}_{5} \mathrm{~N}_{5} \mathrm{O}_{5} \mathrm{H}_{4}\right)_{2}(\mathrm{by})_{2} \cdot 2 \mathrm{by}$ & 300 & 0 & 4.58 & $49.55($ lower $)$ \\
$\mathrm{Fe}\left(\mathrm{C}_{5} \mathrm{~N}_{5} \mathrm{O}_{5} \mathrm{H}_{4}\right)_{3} \cdot 3 \mathrm{DMF} \cdot 1 / 2 \mathrm{H}_{2} \mathrm{O}$ & 272 & 0 & 5.8 & 41.33 (lower) \\
$\mathrm{Co}\left(\mathrm{C}_{5} \mathrm{~N}_{5} \mathrm{O}_{5} \mathrm{H}_{4}\right)_{3} \cdot 3 \mathrm{DMF}$ & 276 & 0 & 5.8 & 65.15 (lower) \\
$\mathrm{Cu}_{4} \mathrm{O}\left(\mathrm{C}_{5} \mathrm{~N}_{5} \mathrm{O}_{5} \mathrm{H}_{3}\right)_{2}\left(\mathrm{CH}_{3} \mathrm{COO}\right)_{2-}$ & 300 & 0 & 4.46 & 71.26 (lower) \\
$\left(\mathrm{CH}_{3} \mathrm{O}\right)_{2} \cdot \mathrm{DMF}$ & & &
\end{tabular}


来看, 多硝基/叠氮基吡啶的合成较为困难, 方法有一定 的局限，部分化合物的结构还不太稳定; 多氨基多硝基 吡啶和苦基硝基吡啶具有较好的热稳定性; 而关于吡啶 类含能离子盐和含能配合物的研究较少, 仅仅作为吡定 类含能化合物研究的补充.

和其他六元含氮芳环相比, 吡啶环电子云密度下降 不多，可被多个硝基取代；又因环上仅有一个氮杂原子， 易于构成对含能化合物稳定性至关重要的对称结构, 这 也是多硝基吡啶近期受到研究者重视的原因, 但与硝基 多氮唑等高氮化合物的研究相比, 吡啶类含能化合物仍 存在一些缺陷, 有必要深入研究以发现新的合成方法或 者化合物结构来适应新的需求, 特别是: (1)研究以简单 易行、绿色环保的合成策略制备全取代的多硝基吡啶类 含能化合物; 对综合性能突出的吡定类炸药, 进行工艺 优化研究, 降低制备成本, 以实现其工业化生产与实际 应用; (2)将吡啶环炸药的钝感性与硝胺或者环脲硝胺的 高能性相结合, 即向吡啶环引入硝胺炸药分子内的同 时, 尽可能多的在炸药分子中引入氮氮键及氮氧键, 从 而合成出高能钝感的吡啶环(嫝)硝胺等新类型炸药; (3) 以不同的吡啶类含能化合物为前体，与高氮有机含能化 合物相组合或与铅、铜等过渡金属离子配位, 来改善和 提高吡啶类含能化合物的总体性能.

\section{References}

[1] Klapötke, T. M.; Penger, A.; Pflüger, C.; Stierstorfer, J.; Sućeska, M. Eur. J. Inorg. Chem. 2013, 2013, 4667.

[2] Zhang, Q.-H.; Zhang, J.-H.; Parrish, D. A.; Shreeve, J. M. Chem. Eur. J. 2013, 19, 11000 .

[3] Fischer, D.; Klapötke, T. M.; Piercey, D. G.; Stierstorfer, J. Chem. Eur. J. 2013, 19, 4602.

[4] Dippold, A. A.; Izsak, D.; Klapötke, T. M. Chem. Eur. J. 2013, 19, 12042.

[5] Dippold, A. A.; Klapötke, T. M. J. Am. Chem. Soc. 2013, 135, 9931.

[6] Millar, R. W.; Philbin, S. P.; Claridge, R. P.; Hamid, J. Propellants, Explos., Pyrotech. 2004, 29, 81.

[7] Gao, H.-X.; Shreeve, J. M. Chem. Rev. 2011, 111, 7377.

[8] Jadhav, H. S.; Talawar, M. B.; Sivabalan, R.; Dhavale, D. D.; Asthana, S. N.; Krishnamurthy, V. N. J. Hazard. Mater. 2007, 143, 192.

[9] Duddu, R.; Dave, P. R.; Damavarapu, R.; Surapaneni, R.; Parrish, D. Synth. Commun. 2009, 39, 4282.

[10] Duddu, R.; Dave, P. R.; Damavarapu, R.; Gelber, N.; Parrish, D. Tetrahedron Lett. 2010, 51, 399

[11] Singh, G.; Kapoor, I. P. S.; Mannan, S. M.; Tiwari, S. K. J. Hazard. Mater. 1999, 68, 155.

[12] Agrawal, J. P. Prop. Explos. Pyrotech. 2005, 30, 316.

[13] Lv, C.-X.; Li, G.-X. Explos. Mater. 1988, 137, 12 (in Chinese). (吕春绪, 李广学, 爆破器材, 1988, (3), 12.)

[14] Zhang, J.-G.; Zhang, T.-L.; Zhang, Z.-G.; Yang, L. Chin. J. Energ. Mater. 2001, 9, 90 (in Chinese). (张建国, 张同来, 张志刚, 杨利, 含能材料, 2001, 9, 90.)

[15] Boddu, V. M.; Viswanath, D. S.; Ghosh, T. K.; Damavarapu, R. J.
Hazard. Mater. 2010, 181, 1.

[16] Carter, J. A.; Zaug, J. M.; Nelson, A. J.; Armstrong, M. R.; Manaa, M. R. J. Phys. Chem. A 2012, 116, 4851.

[17] Nandi, A. K.; Ghosh, M.; Sutar, V. B.; Pandey, R. K. Cent. Eur. J. Energ. Mater. 2012, 9, 119.

[18] Paraskos, A. J.; Kramer, M. P. US 7763753, 2010 [Chem. Abstr 2010, 153, 264293].

[19] Li, J.-S.; Huang, Y.-G.; Dong, H.-S.; Yang, G.-C. Chin. J. Energ. Mater. 2003, 11, 177 (in Chinese).

(李金山，黄奕刚，董海山，杨光成，含能材料, 2003, 11, 177.)

[20] Li, J.-S.; Huang, Y.-G.; Dong, H.-S. Chin. J. Energ. Mater. 2004, 12,576 .

(李金山，黄奕刚，董海山，含能材料, 2004, 12, 576.)

[21] Li, J.-S.; Huang, Y.-G.; Dong, H.-S. J. Energ. Mater. 2005, 23, 133.

[22] Zhao, J.-M.; Li, J.-R.; Wei, X.-J.; Li, W.-T.; Dong, H.-S. Chin. J. Explos. Propellants 2006, 29, 73 (in Chinese).

(赵建民，李加荣，魏篠洁，李文婷，董海山，火炸药学报， 2006, 29, 73.)

[23] Li, J.-R.; Zhao, J.-M.; Dong, H.-S. J. Chem. Crystallogr. 2005, 35, 943.

[24] He, Z.-W.; Gao, D.-Y.; Liu, Z.-L. Chin. J. Explos. Propellants 2009, 32,32 (in Chinese). (何志伟, 高大元, 刘祖亮, 火炸药学报, 2009, 32, 32.)

[25] Zhou, X.-L.; Liu, Z.-L.; Cheng, J.; Su, Q.; Hao, Y.-G.; Hu, B.-C. Chin. J. Energ. Mater. 2013, 21, 423 (in Chinese).

(周心龙, 刘祖亮, 成健, 苏强, 郝尧刚, 胡炳成, 含能材料, 2013, 21, 423.)

[26] Wang, B.-G.; Zhang, J.-L.; Chen, Y.-F. Chin. J. Energ. Mater. 2007, 15, 198 (in Chinese).

(王保国，张景林，陈亚芳，含能材料, 2007, 15, 198.)

[27] Chang, H. Chin. J. Explos. Propellants 2007, 30, 36 (in Chinese). (常海, 火炸药学报, 2007, 30, 36.)

[28] Wan, D.-Z. Chin. Sci. Bull. 1985, 1723 (in Chinese). (万道正，科学通报，1985，1723.)

[29] Licht, H. H.; Ritter, H. Prop. Explos. Pyrotech. 1988, 13, 25.

[30] Bannert, K.; Richter, S.; Schaarschmidt, D.; Lang, H. Angew. Chem., Int. Ed. 2013, 52, 3499.

[31] Huynh, M. V.; Hiskey, M. A.; Hartline, E. L.; Montoya, D. P.; Gilardi, R. Angew. Chem., Int. Ed. 2004, 43, 4924.

[32] Klapötke, T. M.; Piercey, D. G.; Stierstorfer, J. Chem. Eur. J. 2011, 17,13068 .

[33] Huynh, M. H.; Hiskey, M. A.; Chavez, D. E.; Naud, D. L.; Gilardi, R. D. J. Am. Chem. Soc. 2005, 127, 12537.

[34] Crawford, M. J.; Ellern, A.; Mayer P. Angew. Chem., Int. Ed. 2005, 44, 7874.

[35] Zhang, Q.-H.; Shreeve, J. M. Angew. Chem., Int. Ed. 2013, 52, 8792.

[36] Lowe-Ma, C. K.; Nissan, R. A.; Wilson, W. S. J. Org. Chem. 1990, $55,3755$.

[37] VanAllan, J. A.; Reynolds, G. A. J. Heterocycl. Chem. 1968, 5, 471.

[38] Carboni, R. A.; Castle, J. E. J. Am. Chem. Soc. 1962, 84, 2453.

[39] Kauer, J. C.; Carboni, R. A. J. Am. Chem. Soc. 1967, 89, 2633.

[40] Balachari, D.; Trudell, M. L. Tetrahedron Lett. 1997, 38, 8607.

[41] Balachari, D.; Stevens, E. D.; Trudell, M. L.; Beardall, D.; Wight, C. A. Propellants, Explos., Pyrotech. 2000, 25, 75.

[42] Albini, A.; Pietra, S. Heterocyclic N-Oxide, CRC Press, Boca Raton, Florida, 1991

[43] Ritter, H.; Licht, H. H. J. Heterocycl. Chem. 1995, 32, 585.

[44] Cheng, J.; Yao, Q.-Z.; Zhou, X.-L.; Du, Y.; Fang, D.; Liu, Z.-L. Chin. J. Energ. Mater. 2008, 16, 672 (in Chinese). 
(成健，姚其正，周新利，杜杨，方东，刘祖亮，含能材料，2008, 16, 672.)

[45] Cheng, J.; Yao, Q.-Z.; Zhou, X.-L.; Du, Y.; Fang, D.; Liu, Z.-L. Chin. J. Org. Chem. 2008, 28, 1943 (in Chinese).

(成健, 姚其正, 周新利, 杜杨, 方东, 刘祖亮, 有机化学, 2008, 28, 1943.

[46] Cheng, J.; Yao, Q.-Z.; Dong, Y.; Liu, Z.-L. Chin. J. Energ. Mater. 2009, 17, 534 (in Chinese). (成健, 姚其正, 董岩, 刘祖亮, 含能材料, 2009, 17, 534.)

[47] Wang, Y.-H.; Song, Y.-J.; Hu, Z.; Jing, J.-H.; Meng, X.-L.; Huang, Y.-D. Chin. J. Org. Chem. 2009, 29, 780 (in Chinese). (王艳红, 宋元军, 胡桢, 景介辉, 孟祥丽, 黄玉东, 有机化学, 2009, 29, 780.)

[48] Ma, C.-M.; Hou, K.-H.; Liu, Z.-L.; Yao, Q.-Z. Chin. J. Org. Chem. 2014, 34, 584 (in Chinese). (马丛明, 侯可辉, 刘祖亮, 姚其正, 有机化学, 2014, 34, 584.)

[49] Licht, H. H. Propellants, Explos., Pyrotech. 2002, 25, 126.

[50] He, Z.-W.; Cheng, J.; Liu, Z.-L. Chin. J. Energ. Mater. 2009, 17, 392 (in Chinese). (何志伟, 成健, 刘祖亮, 含能材料, 2009, 17, 392.)

[51] He, Z.-W.; Liu, Z.-L.; Wang, A.-L. Chin. J. Explos. Propellants 2010, 22, 11 (in Chinese). (何志伟, 刘祖亮, 王爱玲, 火炸药学报, 2010, 22(1), 11.)

[52] An, C.-W.; Song, X.-L.; Wang, Y.; Guo, X.-D.; Li, F.-S. Chinese J. Energ. Mater. 2007, 15, 188 (in Chinese). (安崇伟, 宋小兰, 王毅, 郭效德, 李风生, 含能材料, 2007, 15, 188.)

[53] Chen, L.-Y.; Zhao, S.-X.; Yang, P.-J.; Heng, S.-Y.; Li, W.; Huang, X.-W. Chin. J. Energ. Mater. 2006, 14, 171 (in Chinese). (陈鲁英, 赵省向, 杨培进, 衡淑云, 李巍, 黄小梧, 含能材料, 2006, 14, 171.)

[54] He, Z.-W.; Gao, D.-Y.; Fang, D.; Liu, Z.-L. Chin. J. Energ. Mater. 2009, 17, 299 (in Chinese).

(何志伟, 高大元, 方东, 刘祖亮, 含能材料, 2009, 17, 299.)

[55] He, Z.-W.; Liu, Z.-L. Chin. J. Explos. Propellants 2013, 36, 21 (in Chinese).

(何志伟, 刘祖亮, 火炸药学报, 2013, 36, 21.)

[56] He, Z.-W.; Liu, Z.-L. Chin. J. Energ. Mater. 2010, 18, 97 (in Chinese).

(何志伟, 刘祖亮, 含能材料, 2010, 18, 97.)

[57] Durante, V. A.; Karmakar, S. US 5861536, 1999 [Chem. Abstr. 1999, 130, 111835].

[58] Bakke, J. M.; Harald, S. Tetrahedron Lett. 2001, 42, 4393.

[59] Cheng, J.; Liu, Z.-L.; Yao, Q.-Z.; Zhou, X.-L.; Wang, A.-L. Chin. J. Explos. Propellants 2009, 32, 5 (in Chinese). (成健, 刘祖亮, 姚其正, 周新利, 王爱玲, 火炸药学报, 2009, $32,5$.

[60] Cheng, J.; Zhou, X.-L.; Qiao, Z.; Yao, Q.-Z.; Liu, Z.-L. Chin. J. Energ. Mater. 2009, 17, 296 (in Chinese).

(成健，周新利，乔珍，姚其正，刘祖亮，含能材料，2009，17, 296.)

[61] Woźniak, M.; Bańskira, A.; Szpakiewicz, B. Liebigs Ann. Chem. $1993,1,7$.

[62] de Bie, D. A.; Geurtsen, B.; van der Plas, H. C. J. Org. Chem. 1985, 50,484 .

[63] Hollins, R. A.; Merwin, L. M.; Nissan, R. A. J. Heterocycl. Chem. 1996, 33, 895

[64] Badgujar, D. M.; Talawar, M. B.; Asthana, S. N.; Mahulikar, P. P. J. Hazard. Mater. 2008, 151, 289

[65] Ritter, H.; Licht, H. H. Propellants, Explos., Pyrotech. 1993, 18 , 81.

[66] Ma, C.-M.; Wang, Y.-B.; Hou K.-H.; Liu, Z.-L.; Yao, Q.-Z. Chin.
J. Chem. 2013, 31, 1299.

[67] Mitchell, A. R.; Pagoria, P. F.; Coon, C. L.; Jessop, E. S.; Poco, J. F.; Tarver, C. M. Breithaupt, R. D.; Moody, G. L. Propellants, Explos., Pyrotech. 1994, 19, 232.

[68] Zhou, C.; Zhou, Y.-S.; Huo, H,; Wang B.-Z.; Zhang, Y.-G. Chin. J. Explos. Propellants 2011, 34, 17 (in Chinese).

(周诚, 周彦水, 霍欢, 王伯周, 张叶高, 火炸药学报, 2011, 34, 17.)

[69] Peng, Z.-J.; Wan, D.-Z. Acta Armament 1980, 3, 23 (in Chinese). (彭忠吉，万道正，兵工学报, 1980, 3, 23.)

[70] Boileau, J.; Emeury, J. L.; Kehren, J. US 4487938, 1984 [Chem. Abstr. 1985, 102, 169227].

[71] Chaykovsky, M.; Adolph, H. G. J. Heterocycl. Chem. 1991, 28 , 1491.

[72] Coburn, M. D. US 3678061, 1972 [Chem. Abstr. 1972, 77, 139812].

[73] Riggs, R. S. US 4948897, 1990 [Chem. Abstr. 1990, 112, 9513].

[74] Wang, N.-X. Chin. J. Explos. Propellants 1993, (4), 21 (in Chinese).

(王乃兴, 火炸药, 1993, (4), 21.)

[75] Wang, N.-X.; Chen, B.-R.; Ou, Y.-X. Chin. J. Explos. Propellants 1992, (4), 6 (in Chinese).

(王乃兴, 陈博仁, 欧育湘, 火炸药, 1992, (4), 6.)

[76] Wang, N.-X. Chin. J. Energ. Mater. 1995, 3, 44 (in Chinese). (王乃兴, 含能材料, 1995, 3, 44.)

[77] Wang, N.-X.; Chen, B.R.; Qu, Y.-X. Chem. World 1993, 34, 254. (王乃兴, 陈博仁, 欧育湘, 化学世界, 1993, 34, 254.)

[78] Wang, N.-X.; Li, J.-S. Chin. J. Energ. Mater. 1994, 2,25 (in Chinese). (王乃兴, 李纪生, 含能材料, 1994, 2, 25.)

[79] Wang, N.-X.; Chen, B.-R.; Ou, Y.-X. J. Beijing Inst. Technol. 1994, 14, 33 (in Chinese). (王乃兴，陈博仁，欧育湘，北京理工大学学报, 1994, 14, 33.)

[80] Coburn, M. D.; Singleton, J. L. J. Heterocycl. Chem. 1972, 9, 1039

[81] Jadhav, H. S.; Talwar, M. B.; Dhavale, D. D.; Asthana, S. N.; Krishnamurthy, V. N. Ind. J. Heterocycl. Chem. 2006, 15, 383.

[82] Singh, R. P.; Verma, R. D.; Meshri, D. T.; Shreeve, J. M. Angew. Chem., Int. Ed. 2006, 45, 3584.

[83] Hong, X.; Arritt, S. W.; Twamley, B.; Shreeve, J. M. Inorg. Chem. 2004, 43, 7972.

[84] Galves-Ruiz, J. C.; Holl, G. H.; Karaghiosoff, K.; Klapötke, T. M.; Löhnwitz, K.; Mayer, P.; Nöth, H.; Polborn, K.; Rohbogner, C. J.; Suter, M.; Weigand, J. J. Inorg. Chem. 2005, 44, 4237.

[85] Thottempudi, V.; Yin, P.; Zhang, J.-H.; Parrish, D. A.; Shreeve, J. M. Chem. Eur. J. 2014, 20, 542.

[86] Klapötke, T. M.; Piercey, D. G.; Stierstorfer, J. Eur. J. Inorg. Chem. 2013, 2013, 1509.

[87] Song, J.-H.; Wang, K.; Liang, L.-X.; Bian, C.-M.; Zhou, Z.-M. RSC Adv. 2013, 3, 10859.

[88] Liang, L.-X.; Cao, D.; Song, J.-H.; Huang, H.-F.; Wang, K.; Bian, C.-M.; Dong, X.; Zhou, Z.-M. J. Mater. Chem. A 2013, 1, 8857.

[89] Gao, H.-X.; Ye, C.-F.; Gupta, O. D.; Xiao, J.; Hiskey, M. A.; Twamley, B.; Shreeve, J. M. Chem. Eur. J. 2007, 13, 3853.

[90] Huang, Y.-G.; Gao, H.-X.; Twamley, B.; Shreeve, J. M. Eur. J. Inorg. Chem. 2007, 2025.

[91] Wang, Y.-L.; Zhao, F.-Q.; Xu, K.-Z.; Ji, Y.-P.; Yi, J.-H.; Chen, B.; An, T. Inorg. Chim. Acta 2013, 405, 505.

[92] Klapötke, T. M.; Mayer, P.; Sabate, C. M.; Welch, J. M.; Wiegand, N. Inorg. Chem. 2008, 47, 6014.

[93] Zhang, G.-F.; She, J. B.; Dou, Y.-L.; Fan, R. Z. Kristallogr.-New Cryst. Struct. 2006, 221, 183.

[94] Tang, F.-Y.; She, J. B.; Zhang, G.-F. Z. Kristallogr.-New Cryst. Struct. 2006, 221, 539 . 
[95] Zheng, Y.-M.; Deng, M.-Z.; Zhao, F.-Q.; Yuan, C. Chin. J. Explos. Propellants 2002, 25, 51 (in Chinese). (郑玉梅, 邓敏智, 赵风起, 袁潮, 火炸药学报, 2002, 25, 51.)

[96] Chen, P.; Zhao, F.-Q.; Luo, Y.; Hu, R.-Z.; Zheng, Y.-M.; Deng, M.-Z.; Gao, Y. Acta Chim. Sinica 2004, 62, 1197 (in Chinese). (陈沛, 赵风起, 罗阳, 胡荣祖, 郑玉梅, 邓敏智, 高茵, 化学学 报, 2004, 62, 1197.)

[97] Zhao, F.-Q.; Chen, P.; Luo, Y.; Zhang, R.-E.; Li, S.-W.; Deng, M.-Z.; Zheng, Y.-M. Chin. J. Explos. Propellants 2003, 26, 1 (in Chinese).

(赵风起, 陈沛, 罗阳, 张苾娥, 李上文, 邓敏智, 郑玉梅, 火炸 药学报, 2003, 26, 1.)

[98] Zhang, G.-F.; She, J.-B.; Li, D.-D.; Li, J.-Z.; Zhou, Q.-P.; Dou, Y.-L.; Fan, X.-Z.; Hu, M.-C.; Liu, Z.-H. J. Coord. Chem. 2008, 61, 865.

[99] Zhou, Q.-P.; Zhang, G.-F.; She, J.-B. J. Coord. Chem. 2008, 61, 2601.

[100] She, J.-B.; Zhang, G.-F.; Zhao, F.-Q.; Lei, Z.-L.; Fan, X.-Z. Struct. Chem. 2007, 18, 373.

[101] Zhang, G.-F.; Yasen·Musa, Zhao, F.-Q.; Fan, X.-Z. Chin. J. Energ. Mater. 2012, 20, 126 (in Chinese).

(张国防, 亚森・木萨, 赵风起, 栎学忠, 含能材料, 2012, 20, 126.)

[102] Cai, M.-Y.; Zhang, G.-F.; Zhou, H.-B.; Jing, P.; He, C. J. Shaanxi Normal Univ. (Nat. Sci. Ed.) 2010, (3), 43 (in Chinese).

(蔡美玉, 张国防, 周海波, 景萍, 何冲, 陕西师范大学学报(自 然科学版), 2010, (3), 43.)

[103] Ghule, V. D.; Srinivas, D.; Muralidharan, K. Asian J. Org. Chem.
2013, 2, 662 .

[104] Zhang, J.-G.; Zhang, T.-L.; Zhang, Z.-G.; Yang, L. Chin. J. Energ. Mater. 2001, 9, 90 (in Chinese).

(张建国, 张同来, 张志刚, 杨利, 含能材料, 2001, 9, 90.)

[105] Liu, J.-J.; Liu, Z.-L.; Lin, X.-Y.; Cheng, J.; Fang, D. Chin. J. Energ. Mater. 2013, 21, 644 (in Chinese).

(刘进剑, 刘祖亮, 﨎向阳, 成健, 方东, 含能材料, 2013, 21, 644.)

[106] Liu, J.-J.; Liu, Z.-L.; Cheng, J.; Fang, D. Chin. J. Energ. Mater. 2013, 21, 711 (in Chinese).

(刘进剑, 刘祖亮, 成健, 方东, 含能材料, 2013, 21, 711.)

[107] Liu, J.-J.; Liu, Z.-L.; Lin, X.-Y.; Cheng, J.; Fang, D. J. Nanjing Univ. Sci. Technol. 2013, 37, 640 (in Chinese).

(刘进剑, 刘祖亮, 萄向阳, 成健, 方东, 南京理工大学学报, 2013, 37, 640.)

[108] Liu, J.-J.; Liu, Z.-L.; Cheng, J.; Fang, D. RSC Adv. 2013, 3, 2917.

[109] Liu, J.-J.; Liu, Z.-L.; Cheng, J. Chin. J. Explos. Propellants 2012, 35, 36 (in Chinese).

(刘进剑，刘祖亮，成健，火炸药学报, 2012, 35, 36.)

[110] Liu, J.-J.; Liu, Z.-L.; Cheng, J. J. Solid State Chem. 2013, 197, 198.

[111] Liu, J.-J.; Liu, Z.-L.; Cheng, J.; Fang, D. J. Solid State Chem. 2013, $200,43$.

[112] Liu, J.-J.; Liu, Z.-L.; Cheng, J.; Fang, D.; Wang, J. Chin. J. Inorg. Chem. 2013, 29, 289.

(刘进剑, 刘祖亮, 成健, 方东, 王俊, 无机化学学报, 2013, 29, 289.)

[113] Yamanouchi, K.; Enemark, J. H. Inorg. Chem. 1978, 17, 2911.

(Qin, X.) 